\title{
Evaluating Slow Pyrolysis of Parthenium hysterophorus Biochar: Perspectives to Acidic Soil Amelioration and Growth of Selected Wheat (Triticum aestivum) Varieties
}

\author{
Meseret Muche $\mathbb{D}^{1},{ }^{1}$ Eyayu Molla, ${ }^{2}$ Sultan Mohammed, ${ }^{1}$ Esubalew Sintie, ${ }^{1}$ \\ and Ahmed Hassen ${ }^{1}$ \\ ${ }^{1}$ Department of Biology, Woldia University, P.O. Box 400, Woldia, Ethiopia \\ ${ }^{2}$ Department of Natural Resource Management, College of Agriculture and Environmental Sciences, Bahir Dar University, \\ Bahir Dar, Ethiopia
}

Correspondence should be addressed to Meseret Muche; muchemeseret@wldu.edu.et

Received 25 August 2021; Revised 26 November 2021; Accepted 29 November 2021; Published 4 January 2022

Academic Editor: Cezary Slawinski

Copyright (c) 2022 Meseret Muche et al. This is an open access article distributed under the Creative Commons Attribution License, which permits unrestricted use, distribution, and reproduction in any medium, provided the original work is properly cited.

\begin{abstract}
Application of biochar on acidic soils may improve soil fertility and crop productivity. This study aimed to explore the relevance of parthenium biochar-induced changes in the physicochemical properties and agronomic performance of the selected wheat varieties in acidic soils. A pot trial was used in determining the effect of slow pyrolysis parthenium biochar on acidic soils and the agronomic performance of wheat varieties. A general linear model (GLM) of multivariate analysis and principal component analysis (PCA) was used to compare functional variation among soil assayed parameters with biochar dosages and years. Biochartreated acidic soils did not show significant differences in their physical properties. However, a significant incremental trend was observed in the soil moisture content. The biochar-amended acidic soils showed noticeable differences in the soil $\mathrm{pH}$, available phosphorous, and exchangeable bases $(\mathrm{Ca}, \mathrm{K}$, and $\mathrm{Na}$ ) compared to the control. In all soil samples, a decreasing trend in the soil micronutrients was observed with an increase in the biochar amounts. The analysis also unveiled significant changes in root length, root and shoot dry biomass, and plant height of wheat varieties in response to the biochar amendments. The application of $19.5 \mathrm{t} / \mathrm{ha}$ and $23 \mathrm{t} / \mathrm{ha}$ dosages of biochar gave the maximum changes in the agronomic performance of Kekeba and Ogolcha varieties, while the minimum was obtained in the $26.5 \mathrm{t} / \mathrm{ha}$ and the control. Furthermore, PCA axis 1 accounted for $74.34 \%$ of the total variance within a higher eigenvector value (10.4076), and most of the soil parameters were positively correlated with CEC (0.29), available phosphorous (0.29), and soil $\mathrm{pH}(0.28)$; however, the micronutrients were negatively correlated. In conclusion, Parthenium hysterophorus biochar has the potential to amend acidic soils, and thus, the application of 16.0, 19.5, and $23 \mathrm{t} \cdot \mathrm{ha}^{-1}$ biochar dosages are considered suitable to reduce the soil acidity level and improve the agronomic performance of wheat varieties. However, extensive research will be needed to determine the effects of biochar on soil properties and crop production in field conditions.
\end{abstract}

\section{Introduction}

In Ethiopia, low soil fertility is one of the factors limiting the yield of many crops. This is caused by the removal of surface soil by erosion, crop removal of nutrients from the soil, total removal of plant residue from farmland, and lack of proper crop rotation practices $[1,2]$. On the other hand, invasive herbaceous weedy species such as Parthenium hysterophorus are increasing in different regions of Ethiopia [3]. $P$. hysterophorus (Asteraceae) is an aggressive alien weed species native to the Americas, and at present, it is extensively spread in Asia, Australia, and Africa [4] and Ethiopia [3]. It grows along roadsides and in fallow and cultivated lands, riverbanks, disturbed areas, and floodplains. It competes with and replaces native species and is also a significant crop weed [3-5]. It is the most noxious weed in 
the agricultural system due to prolific seed production, allelopathic effect, and competitiveness [5]. Various studies have been conducted on the toxic effect of the $P$. hysterophorus on a wide range of crops. For instance, studies showed that extracts of the weed at lower concentrations (2.5-4\%) significantly diminished seed germination, seedling biomass, and chlorophyll content of wheat by $60-75 \%[6,7]$ and grain yields of sorghum and maize by $40-97 \%$ and $30-60 \%$, respectively [5]. However, different studies proposed that parthenium biochar can be used as green manure, compost, biocontrol, and soil amelioration that improve the soil physical, chemical, and biological properties and is a source of readily available plant microand macronutrients [8-10]. Hence, amendment of soil acidity through pyrolysis carbon extracted biochar from this aggressive weed ( $P$. hysterophorus) is momentous. Biochar (BC) is a fine-grained carbon-rich product obtained when the biomass is heating in an oxygen-depleted atmosphere $[11,12]$. It contains porous carbonaceous and an array of functional groups [13]. Recently, the potential of biochar use to recapture excess soil nutrients, crops, and remove contaminants has received growing attention [14-17]. The feedstock sources and pyrolytic temperatures are the principal factors for the nutrient provisions. And thence, the herbaceous feedstock may pyrolyze above $400^{\circ} \mathrm{C}$ and woody raw materials even above $800^{\circ} \mathrm{C}$ [18]. The addition of biochar to acidic soils changes soil $\mathrm{pH}$ from 3.9 to 5.1 [19], boost electrical conductivity [20], boost cation exchange capacity from 7.41 to $10.8 \mathrm{cmol}^{+} / \mathrm{kg}$ [21], and increase the percent base saturation from 6.4 to $26 \%$ and modifies soil acidity [22]. Therefore, the present study was initiated to assess the potential effect of $P$. hysterophorus biochar on the amelioration of acidic soils and the agronomic performance of selected wheat (Triticum aestivum) crop varieties.

\section{Materials and Methods}

2.1. Description of the Study Area. The study was conducted at the research site of Woldia University, Northeastern Ethiopia, from June 2018 to November 2020. Geographically, the site lies between $11^{\circ} 35^{\prime}$ and $12^{\circ} 00^{\prime} \mathrm{N}$ latitude and $39^{\circ} 14^{\prime}$ and $39^{\circ} 48^{\prime}$ E longitude and 2,740 meters above sea level (masl). The mean annual rainfall recorded during the study period was $1,050 \mathrm{~mm}$, and the average annual minimum and maximum temperatures were 18 and $28.7^{\circ} \mathrm{C}$, respectively [23]. The district was a representative of wet highland and characterized by erosion-prone, susceptible to acidity, low potential, and oxen plow cereal belt area. The main crop types grown in the district are oats (Avena sativa L.), line seed (Linum usitatissimum L.), barley (Hordeum vulgare L.), and wheat (T. aestivum). The area was selected for the study because soil acidity and invasion of parthenium weed in the area are important issues that require urgent attention.

2.2. Preparation of the Biochar Component. Fresh $P$. hysterophorus weed was collected in the vicinity of the study site before flowering to prevent seed dispersal. The entire plant material (biomass) was washed with distilled water to remove impurities and allowed to air-dry. The dried weed was cut down into smaller pieces $(10-15 \mathrm{~cm})$ and treated with an active chemical $\left(7 \% \mathrm{H}_{2} \mathrm{SO}_{4} / 1 \mathrm{~kg}\right)$ to lower the temperature of carbonization [24]. A kilogram of chemically treated dried sample was tightly placed in a closed perforated austenitic stainless steel covered with a fitting lid and inserted into the muffle furnace and then charred at $350^{\circ} \mathrm{C}$ (slow pyrolysis temperature) for 30 minutes in an oxygenfree medium [25]. The biochar produced was transferred from the muffle furnace into the pot (height of $40.0 \mathrm{~cm}$ and $27.0 \mathrm{~cm}$ width) and washed thoroughly with distilled water to remove the component of chemically active acid. At the end, the recommended dosages of fine-grained biochar was characterized (Table 1; Supplementary 1), measured [26], and denoted based on the dose of parthenium biochar (PB) as PB0\% (0 t/ha), PB5.33\% (12.5 t/ha), PB8.0\% (16.0 t/ha), PB10.67\% (19.5 t/ha), PB13.3\% (23t/ha), and PB16\% (26.5 t/ ha). The pyrolyzed biochar was later grounded and sieved with a $0.05 \mathrm{~mm}$ sieve and made ready for application.

\subsection{Research Design, Soil Analysis, and Crop Data Collection}

2.3.1. Treatments and Experimental Design. In this study, a pot experimental design that involves a complete randomized design (CRD) with three replications of three (wheat varieties) $\times$ six (biochar rates) factorial combinations was used. Before the experiment, the $\mathrm{pH}$ of the soil samples (ranging from 5.2 to 5.6) was randomly collected at $0-25 \mathrm{~cm}$ depth from 15 pits of acidic farmlands. The soil samples were bulked together to serve as composite soil samples. Then a $12 \mathrm{~kg}$ of dry acidic soil was mixed with different dosages of biochar filled in each $40 \mathrm{~cm}$ height and 27.0 widths experimental pot. The $P$. hysterophorus biochar dosages were $0,12.5,16.0,19.5,23$, and $26.5 \mathrm{t} / \mathrm{ha}$, which accounted for the dry weights of the potting soil (Supplementary 2). The pots were left for two months with three days interval of $2000 \mathrm{~mL}$ watering for decomposition and merely mixed the biochar into the acidic soil $[26,27]$. After preparing the pots filled with equal amounts of soil, different amount of biochar was added as per treatments in a complete randomized design with three replications. Three wheat varieties (Kekeba, Ogolcha, and Kingbird) obtained from Ethiopia Agricultural Research Institute were used as test crops. Before sowing, the seeds were first washed with distilled water and sterilized with $2 \%$ sodium hypochlorite for 2 minutes. Accordingly, pots were filled with equal amounts of soil and different dosages of biochar and arranged into blocks, and one of the treatments was used as a control (Supplementary 2). Afterward, 15 viable seeds were selected and evenly sowed into each pot, and each germinating pot was regularly supplied with $2,000 \mathrm{~mL}$ of water once a day in the morning. Cultural practices such as weeding, hoeing, disease, and pest control were applied uniformly for all treatments to produce healthy and pure seedlings. Weeds were managed by hand weeding after weed emergence. Finally, the wheat varieties were separately harvested. 
TABLE 1: The chemical composition of the acidic soil sample and the parthenium biochar.

\begin{tabular}{lccccccc}
\hline Chemical Properties & $\begin{array}{c}\mathrm{pH} \\
\left(\mathrm{H}_{2} \mathrm{O}\right)\end{array}$ & $\begin{array}{c}\mathrm{EC} \\
\mathrm{ms} / \mathrm{cm}\end{array}$ & $\begin{array}{c}\mathrm{OC} \\
(\%)\end{array}$ & $\begin{array}{c}\mathrm{CEC} \\
(\mathrm{Meq} / 100 \mathrm{~kg})\end{array}$ & $\begin{array}{c}\text { Ex. Ca } \\
\left(\mathrm{cmol}^{+} / \mathrm{kg}\right)\end{array}$ & $\begin{array}{c}\text { Ex. K } \\
\left(\mathrm{cmol}^{+} / \mathrm{kg}\right)\end{array}$ & $\begin{array}{c}\text { Av. P } \\
(\mathrm{ppm})\end{array}$ \\
\hline Acidic soil sample & 5.4 & 0.12 & 0.8 & 20.2 & 11.5 & 0.6 & 13.8 \\
Parthenium biochar & 12.23 & 10.7 & 26.8 & 42.64 & 25.6 & 15.4 & 176.7 \\
\hline
\end{tabular}

Note. $\mathrm{pH}$, power of hydrogen; EC, electrical conductivity; OC, organic carbon; CEC, cation exchange capacity; Ex.Ca, exchangeable calcium; Ex. K, exchangeable potassium; and Av. P, available phosphorous.

2.3.2. Soil Analysis. Soil samples were collected before and after treatments (Table 1) in the farmlands and analyzed following the standard laboratory protocols. Soil particle size was analyzed following the hydrometer or Bouyoucos method [28]. Soil moisture content was determined by the percentage weight loss of the soil sample after being dried at $105^{\circ} \mathrm{C}$ divided by the dry soil weight [29]. The soil $\mathrm{pH}$ was measured in water $\left(\mathrm{pH}\left(\mathrm{H}_{2} \mathrm{O}\right)\right)$ and potassium chloride $(1 \mathrm{M}$ $\mathrm{KCl}$ ) [28]. Soil organic carbon content was analyzed by wet combustion or dichromate oxidation methods [30]. The soil available phosphorous content was determined by the $0.5 \mathrm{M}$ sodium bicarbonate extraction solution/pH 8.5/method of Olsen as described by [20]. Exchangeable basic cations $\left(\mathrm{Ca}^{2+}, \mathrm{Mg}^{2+}, \mathrm{K}^{+}\right.$, and $\left.\mathrm{Na}^{+}\right)$were analyzed by saturating the soil samples with $1 \mathrm{~N} \mathrm{NH}_{4} \mathrm{OAc}$ solution at $\mathrm{pH}$ 7. Then $\mathrm{Ca}^{2+}$ and $\mathrm{Mg}^{2+}$ were determined from the extract using atomic absorption spectrometry (AAS), while exchangeable $\mathrm{K}^{+}$and $\mathrm{Na}^{+}$were determined using a flame photometer from the same extracted [31]. Cation exchange capacity (CEC) was estimated titrimetrically by distillation of ammonium displaced by sodium from $\mathrm{NaCl}$ solution [32]. Furthermore, the available micronutrients $(\mathrm{Cu}, \mathrm{Mn}, \mathrm{Zn}$, and $\mathrm{Fe}$ ) were measured after extraction with $1 \mathrm{M} \mathrm{NH}_{4} \mathrm{OAc}$ as described by [31].

2.3.3. Agronomic Data Collection. The following phonological crop data were collected in each wheat variety as recommended by [33]. The assay parameters were plant height $(\mathrm{PH})$, head length $(\mathrm{HL})$, spike number $(\mathrm{SN})$, seed number per plant (SNPP), root length (RL), shoot dry biomass (SDB), and root dry biomass (RDB). Plant height $(\mathrm{PH} ; \mathrm{cm})$ was recorded from randomly selected ten wheat plants and measured from the soil surface to the top-most growth point of plants at the time of physiological maturity, and the mean value was used for analysis. Head length (HL; $\mathrm{cm}$ ) was recorded from randomly selected ten wheat plants from the uppermost part of the peduncle to the tip of grainbearing parts at maturity, and the mean value was taken for analysis. Spikes number (SN) was counted from ten randomly taken wheat plants at physiological maturity in length, and then average value was recorded. Seed number per plant (SNPP) was measured by counting the number of grains per spike in each experimental pot at harvesting time. Root length (RL; $\mathrm{cm}$ ) was measured lengthwise from the crown (underneath the ground where the secondary roots emerge) to the tip of the primary root at harvesting time after properly uprooted from the experimental pot. Shoot dry biomass (SDB; gram) was measured from ten randomly selected wheat plants from the net pot area at the time of harvesting, and then the samples were air-dried for 72 hours, after which weight was taken. Root dry biomass (RDB; gram) was also recorded by taking the average below-ground biomass of ten randomly selected wheat plants after the samples were air drying out for 72 hours.

2.4. Data Analysis. The data were subjected to multivariate analysis (two- and three-way ANOVA) using the general linear model (GLM) procedures of SAS v.9.1.3 to compare the soil physicochemical properties and growth of the varieties influenced by parthenium weed biochar across the soil sample years. Mean comparisons were employed using least significant difference (LSD) at 5\% levels. The principal component analysis (PCA) was used for determining the functional variation of the soil assayed parameters after the data log-transformed using PAST version 3.0 statistical analysis software.

\section{Results}

The biochar made from the P. hysterophorus weed improves the soil quality and increases the growth of the selected wheat varieties. Thus, parthenium biochar is characterized by higher $\mathrm{pH}, \mathrm{C}$ content, exchangeable bases, and available phosphorus content (Table 1), which amends acidity in the soil system.

3.1. Influence of P. hysterophorus Biochar on the Physical Properties of Acidic Soil. Soil particle distribution did not show a significant $(p>0.05)$ difference between the soils sampled in different years (Table 2). Similarly, there was no significant difference between the interaction effect of sand $\left(F(5,35)=2.3 ; p>0.05 ; R^{2}=0.84\right)$, clay $(F(5,35)=1.7$; $\left.p>0.05 ; R^{2}=0.72\right)$, and silt particles contents $(F(5,35)=2.1$; $p>0.05 ; R^{2}=0.52$; Table 2$)$. Conversely, there was an increase in the clay $(p \leq 0.001)$ and silt $(p \leq 0.05)$ contents. However, a significant reduction in the sand fraction $(p \leq 0.001)$ was observed with an increase in the dose of mixed biochar (Tables 2 and 3). The post hoc test in Table 3 revealed a higher overall mean of the sand fraction $(61.3 \pm 1.0)$ was recorded in the control $(0 \mathrm{t} / \mathrm{ha})$, and however, the clay $(23.0 \pm 0.7)$ and silt $(20.5 \pm 0.00)$ contents were observed in $16.0 \mathrm{t} / \mathrm{ha}$ and $26.5 \mathrm{t} / \mathrm{ha}$ biochar rates, respectively. The soil moisture content (SMC) showed a consistent and significant $(p \leq 0.01)$ change among the soils treated with different dosages of $P$. hysterophorus biochar and soil sample years $(p \leq 0.05)$. However, the interaction effect $(F$ $\left.(5,35)=1.98 ; p>0.05 ; R^{2}=0.95\right)$ between the soil sample years and biochar application was insignificant (Table 2). 
TABLE 2: Results of the general linear model procedure, analyzing the effect of parthenium biochar dosages and soil sample years on the soil physical properties.

\begin{tabular}{|c|c|c|c|c|c|c|c|c|c|}
\hline \multirow{2}{*}{ Sources of variations } & \multirow{2}{*}{$\mathrm{DF}$} & \multicolumn{2}{|c|}{ Sand (\%) } & \multicolumn{2}{|c|}{ Clay (\%) } & \multicolumn{2}{|c|}{ Silt (\%) } & \multicolumn{2}{|c|}{ SMC (\%) } \\
\hline & & MS & $\mathrm{P}$ & MS & $\mathrm{P}$ & MS & $\mathrm{P}$ & MS & $\mathrm{P}$ \\
\hline SSY & 1 & 2.8 & $p>0.05$ & 2.2 & $p>0.05$ & 0.2 & $p>0.05$ & 0.035 & $p \leq 0.05$ \\
\hline PBD & 5 & 15.2 & $p \leq 0.01$ & 7.1 & $p \leq 0.01$ & 4.2 & $p \leq 0.05$ & 30.06 & $p \leq 0.01$ \\
\hline $\mathrm{SSY}^{*} \mathrm{PBD}$ & 5 & 1.6 & $p>0.05$ & 1.2 & $p>0.05$ & 2.98 & $p>0.05$ & 0.40 & $p>0.05$ \\
\hline Error & 24 & 0.69 & - & 0.69 & - & 1.42 & - & 0.3 & - \\
\hline Mean & & 58.4 & - & 22.02 & - & 19.7 & - & 19.13 & - \\
\hline$R$-square $\left(R^{2}\right)$ & & 0.84 & - & 0.72 & - & 0.52 & - & 0.95 & - \\
\hline Total & 36 & 3412.4 & - & 486.6 & - & 390.0 & - & 370.32 & - \\
\hline
\end{tabular}

Note. SSY, soil sample years; PBD, parthenium biochar dosages; MS, mean square; P, probability level; DF, degree of freedom, and SMC, soil moisture content.

TABLE 3: Effect of P. hysterophorus biochar amounts $(n=6)$ and years $(n=2)$ on the soil texture and SMC of acidic soils (mean \pm SD).

\begin{tabular}{|c|c|c|c|c|c|c|c|c|}
\hline Soil & Soil sample & & & Partheniur & biochar rates & & & \\
\hline Parameters & Years & $\begin{array}{l}\text { PB0\% } \\
0 \mathrm{t} / \mathrm{ha}\end{array}$ & $\begin{array}{l}\text { PB5.33\% } \\
12.5 \text { t/ha }\end{array}$ & $\begin{array}{c}\text { PB8.0\% } \\
16 \mathrm{t} / \mathrm{ha}\end{array}$ & $\begin{array}{c}\text { PB10.67\% } \\
19.5 \text { t/ha }\end{array}$ & $\begin{array}{c}\text { PB13.3\% } \\
23 \text { t/ha }\end{array}$ & $\begin{array}{l}\text { PB 16\% } \\
26.5 \text { t/ha }\end{array}$ & LSD \\
\hline Sand & $\begin{array}{c}\text { First year } \\
\text { Second year } \\
\text { Overall }\end{array}$ & $\begin{array}{c}60.61 \pm 0.6^{\mathrm{a} *} \\
61.9 \pm 1.1^{\mathrm{a} *} \\
61.3 \pm 1.0^{\mathrm{a} *}\end{array}$ & $\begin{array}{c}57.6 \pm 0.6^{\mathrm{c}} \\
57.3 \pm 1.0^{\mathrm{bc}} \\
57.5 \pm 0.7^{\mathrm{c}}\end{array}$ & $\begin{array}{l}58.9 \pm 0.6^{\mathrm{bc}} \\
57.3 \pm 1.0^{\mathrm{bc}} \\
58.1 \pm 1.1^{\mathrm{dc}}\end{array}$ & $\begin{array}{c}57.6 \pm 1.1^{\mathrm{c}} \\
56.9 \pm 0.6^{\mathrm{bc}} \\
57.3 \pm 0.8^{\mathrm{c}}\end{array}$ & $\begin{array}{l}57.6 \pm 0.5^{\mathrm{c}} \\
56.6 \pm 0.6^{\mathrm{c}} \\
57.1 \pm 0.7^{\mathrm{c}}\end{array}$ & $\begin{array}{l}59.6 \pm 0.6^{\mathrm{a}} \\
58.6 \pm 0.6^{\mathrm{b}} \\
58.1 \pm 0.9^{\mathrm{b}}\end{array}$ & $\begin{array}{c}3.0 \\
0.77 \\
0.42 \\
\end{array}$ \\
\hline Clay & $\begin{array}{c}\text { First year } \\
\text { Second year } \\
\text { Overall } \\
\end{array}$ & $\begin{array}{c}20.2 \pm 0.00^{\mathrm{c}} \\
21.2 \pm 1.0^{\mathrm{c}} \\
20.6 \pm 0.8^{\mathrm{b}} \\
\end{array}$ & $\begin{array}{l}23.5 \pm 0.6^{\mathrm{a} *} \\
22.5 \pm 0.6^{\mathrm{bc}} \\
23.0 \pm 0.7^{\mathrm{a} *} \\
\end{array}$ & $\begin{array}{c}22.8 \pm 1.1^{\mathrm{ab}} \\
23.2 \pm 1.0^{\mathrm{ab} * *} \\
23.0 \pm 0.9^{\mathrm{a} *}\end{array}$ & $\begin{array}{c}21.5 \pm 1.1^{\mathrm{bc}} \\
23.2 \pm 1.0^{\mathrm{ab} * *} \\
22.3 \pm 1.3^{\mathrm{a}}\end{array}$ & $\begin{array}{c}22.2 \pm 0.00^{\mathrm{ab}} \\
22.8 \pm 0.6^{\mathrm{ab}} \\
22.5 \pm 0.5^{\mathrm{a}} \\
\end{array}$ & $\begin{array}{c}20.5 \pm 0.6^{\mathrm{c}} \\
20.8 \pm 1.1^{\mathrm{c}} \\
20.67 \pm 0.8^{\mathrm{b}} \\
\end{array}$ & $\begin{array}{l}3.0 \\
2.3 \\
2.3 \\
\end{array}$ \\
\hline Silt & $\begin{array}{c}\text { First year } \\
\text { Second year } \\
\text { Overall } \\
\end{array}$ & $\begin{array}{c}19.2 \pm 0.6 \\
17.2 \pm 1.1^{\mathrm{b}} \\
18.2 \pm 1.3^{\mathrm{b}} \\
\end{array}$ & $\begin{array}{c}18.9 \pm 0.6 \\
20.2 \pm 0.6^{\mathrm{a}} \\
19.5 \pm 0.9^{\mathrm{ab}} \\
\end{array}$ & $\begin{array}{c}18.5 \pm 2.0 \\
20.2 \pm 1.5^{\mathrm{a}} \\
19.4 \pm 1.8^{\mathrm{ab}}\end{array}$ & $\begin{array}{c}20.9 \pm 2.3 \\
19.9 \pm 1.1^{\mathrm{a}} \\
20.4 \pm 1.7^{\mathrm{a}} \\
\end{array}$ & $\begin{array}{c}20.2 \pm 0.6 \\
20.5 \pm 0.00^{\mathrm{a} * *} \\
20.4 \pm 0.4^{\mathrm{a}} \\
\end{array}$ & $\begin{array}{c}19.9 \pm 1.1 \\
20.5 \pm 0.0^{\mathrm{a} * *} \\
20.5 \pm 0.0^{\mathrm{a} * *} \\
\end{array}$ & $\begin{array}{c}\text { NS } \\
0.77 \\
2.2 \\
\end{array}$ \\
\hline$\% \mathrm{SMC}$ & $\begin{array}{c}\text { First year } \\
\text { Second year } \\
\text { Overall }\end{array}$ & $\begin{array}{l}15.8 \pm 0.3^{\mathrm{e}} \\
15.1 \pm 0.2^{\mathrm{d}} \\
15.5 \pm 0.5^{\mathrm{e}}\end{array}$ & $\begin{array}{c}17.5 \pm 0.4^{\mathrm{cd}} \\
17.99 \pm 0.3^{\mathrm{c}} \\
17.7 \pm 0.4^{\mathrm{d}}\end{array}$ & $\begin{array}{c}18.99 \pm 0.2^{\mathrm{bc}} \\
18.8 \pm 0.9^{\mathrm{c}} \\
19.4 \pm 0.8^{\mathrm{c}}\end{array}$ & $\begin{array}{l}19.4 \pm 0.8^{\mathrm{b}} \\
20.1 \pm 0.6^{\mathrm{b}} \\
19.8 \pm 0.8^{\mathrm{b}}\end{array}$ & $\begin{array}{c}20.8 \pm 0.8^{\mathrm{a}} \\
20.99 \pm 0.4^{\mathrm{ab}} \\
20.9 \pm 0.6^{\mathrm{a}}\end{array}$ & $\begin{array}{c}21.02 \pm 0.4^{\mathrm{a} *} \\
21.9 \pm 0.1^{\mathrm{a} *} \\
21.6 \pm 0.5^{\mathrm{a} *}\end{array}$ & $\begin{array}{l}1.98 \\
2.61 \\
1.82\end{array}$ \\
\hline
\end{tabular}

Within rows, means followed by the same letter are not significantly different $(p>0.05) ;{ }^{*}$ significant at $p \leq 0.01 ;{ }^{* *}$ significant at $p \leq 0.05$; NS, not significant; LSD, least significant difference; \% SMC, percent soil moisture content; PB; parthenium biochar; and SD, standard deviation.

Irrespective of the interaction effect, the soil water content was increasingly higher as biochar amounts increased to result in a slight increase in the overall mean soil moisture content $(21.6 \pm 0.5)$ in the soil with a $26.5 \mathrm{t} / \mathrm{ha}(16 \%)$ $P$. hysterophorus biochar dose compared to the control (15.5 \pm 0.5; Table 3).

3.2. Chemical Properties of Acidic Soil Treated by $P$. hysterophorus Biochar. Soil $\mathrm{pH}$ showed a statistically significant difference $(p \leq 0.01)$ between the biochar dosages across the soil sample years (Table 4). Regardless of the interaction effects, there was an increasing trend in the soil $\mathrm{pH}$ values with the pyrolysis weed (Table 5). The highest soil $\mathrm{pH}\left(\mathrm{H}_{2} \mathrm{O}: 7.8 \pm 0.5\right.$ and $\left.\mathrm{KCl}: 7.1 \pm 0.5\right)$ was recorded in the soil treated with $26.5 \mathrm{t} /$ ha biochar, while the lowest was observed in the control (Table 5). In addition, a significant $(p \leq 0.001)$ increase in EC content occurred as biochar concentration increased within the soil sample years $(F(5$, $35)=10.07 ; p \leq 0.001 ; R^{2}=0.97$; Table 5). Application of a $26.5 \mathrm{t} / \mathrm{ha}$ of biochar increased the soil EC by 14.88 and $12.33 \%$ as compared to the control in the first and second soil sample years, respectively. Similarly, biochar-treated acidic soils across the soil sample years showed significant $(p \leq 0.001)$ changes in exchangeable bases $(\mathrm{Ca}, \mathrm{K}$, and $\mathrm{Na})$ and CEC (Table 4). In addition, there was a significant interaction effect between exchangeable $\mathrm{Ca}^{++}(F(5,35)=5.8$; $\left.p \leq 0.005 ; \quad R^{2}=0.90\right), \quad \mathrm{Na}^{+}(F(5,35)=5.4 ; \quad p \leq 0.005 ;$ $\left.R^{2}=0.81\right)$, and $\operatorname{CEC}\left(F(5,35)=31.4 ; p \leq 0.005 ; R^{2}=0.92\right.$; Table 4). The higher overall average values of the exchangeable bases and CEC found in the P. hysterophorus biochar ranged between 23 and $26.5 \mathrm{t} / \mathrm{ha}$ (Table 5). On the contrary, the exchangeable $\mathrm{Mg}^{2+}$ content was not influenced by the biochar dosages $(p>0.05)$ and with the interaction effect (Table 4 ). Despite the interaction effect, a maximum $\left(7.3 \pm 2.0 \mathrm{cmol}^{+} / \mathrm{kg}\right)$ value was recorded in the soil treated with $16 \mathrm{t} /$ ha of biochar and the minimum in control (Table 5). The contents of available phosphorous and soil organic carbon also showed a considerable increase with the increase in biochar amounts and soil sample years (Table 4). As a result, the highest Av. P (73.2 $\pm 9.2 \mathrm{ppm})$ and SOC $(2.2 \pm 0.5 \%)$ contents found in the soils treated with $26.5 \mathrm{t} / \mathrm{ha}$ biochar that raised the Av. P content up to $5.75 \%$ and SOC by $3.6 \%$ compared to the control (Table 5 ).

The multivariate analysis also revealed a significant difference $(p \leq 0.001)$ in the contents of soil micronutrients between biochar-treated soils, but not in the interaction effect of the soil sample years and the amounts (Table 6). Irrespective of the significant difference in the interaction effect, there was a consistent decrease in the amounts of 


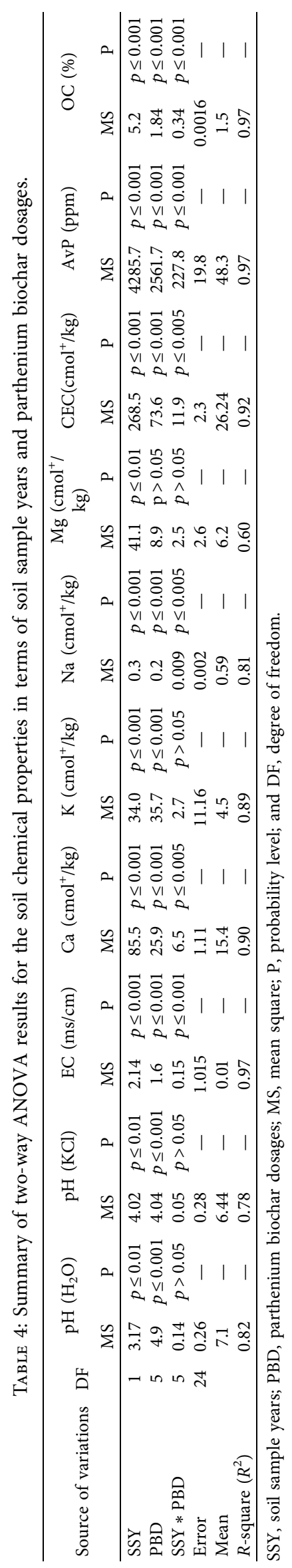


TABLE 5: The status of chemical properties in acidic soil into two soil sample years and parthenium biochar dosages (mean \pm SD).

\begin{tabular}{|c|c|c|c|c|c|c|c|c|}
\hline \multirow[b]{2}{*}{ Soil parameters } & \multicolumn{3}{|l|}{ Soil sample } & \multicolumn{3}{|c|}{ Parthenium biochar rates } & \multirow[b]{2}{*}{$\begin{array}{l}\text { PB16\% } \\
26.5 \text { t/ha }\end{array}$} & \multirow[b]{2}{*}{ LSD } \\
\hline & Years & $\begin{array}{l}\text { PB0\% } \\
0 \text { t/ha }\end{array}$ & $\begin{array}{l}\text { PB5.33\% } \\
12.5 \mathrm{t} / \mathrm{ha}\end{array}$ & $\begin{array}{c}\text { PB8.0\% } \\
16 \text { t/ha }\end{array}$ & $\begin{array}{c}\text { PB10.67\% } \\
19.5 \text { t/ha }\end{array}$ & $\begin{array}{c}\text { PB13.3\% } \\
23 \text { t/ha }\end{array}$ & & \\
\hline $\mathrm{pH}\left(\mathrm{H}_{2} \mathrm{O}\right)$ & $\begin{array}{l}\text { First year } \\
\text { Second year } \\
\text { Combined }\end{array}$ & $\begin{array}{l}5.44 \pm 0.12^{\mathrm{b}} \\
5.45 \pm 0.14^{\mathrm{d}} \\
5.44 \pm 0.12^{\mathrm{c}}\end{array}$ & $\begin{array}{l}6.3 \pm 0.5^{\mathrm{ab}} \\
7.0 \pm 0.2^{\mathrm{c}} \\
6.7 \pm 0 .^{5 \mathrm{~b}}\end{array}$ & $\begin{array}{l}6.7 \pm 0.5^{\mathrm{a}} \\
7.6 \pm 0.5^{\mathrm{b}} \\
7.2 \pm 0.7^{\mathrm{ba}}\end{array}$ & $\begin{array}{c}7.1 \pm 1.1^{\mathrm{a}} \\
7.96 \pm 0.2^{\mathrm{ab}} \\
7.55 \pm 0.8^{\mathrm{a}}\end{array}$ & $\begin{array}{c}7.5 \pm 0.6^{\mathrm{a} * *} \\
8.1 \pm 0.2^{\mathrm{a} *} \\
7.8 \pm 0.5^{\mathrm{a} * *}\end{array}$ & $\begin{array}{c}7.3 \pm 0.6^{\mathrm{a}} \\
8.1 \pm 0.15^{\mathrm{a}} \\
7.8 \pm 0.5^{\mathrm{a}}\end{array}$ & $\begin{array}{l}2.06 \\
1.58 \\
1.74 \\
\end{array}$ \\
\hline $\mathrm{pH}(\mathrm{KCl})$ & $\begin{array}{l}\text { First year } \\
\text { Second year } \\
\text { Combined }\end{array}$ & $\begin{array}{c}4.8 \pm 0.05^{\mathrm{b}} \\
5.2 \pm 0.7^{\mathrm{c}} \\
5.0 \pm 0.5^{\mathrm{c}}\end{array}$ & $\begin{array}{c}5.64 \pm 0.5^{\mathrm{a}} \\
6.3 \pm 0.1^{\mathrm{b}} \\
5.99 \pm 0.5^{\mathrm{b}}\end{array}$ & $\begin{array}{c}6.1 \pm 0.6^{\mathrm{a}} \\
6.9 \pm 0.5^{\mathrm{a}} \\
6.5 \pm 0.7^{\mathrm{ab}}\end{array}$ & $\begin{array}{c}6.4 \pm 1.0^{\mathrm{a}} \\
7.3 \pm 0.13^{\mathrm{a}} \\
6.9 \pm 0.8^{\mathrm{a}}\end{array}$ & $\begin{array}{c}6.8 \pm 0.6^{\mathrm{a} * *} \\
7.4 \pm 0.1^{\mathrm{a} * *} \\
7.1 \pm 0.5^{\mathrm{a}}\end{array}$ & $\begin{array}{c}6.8 \pm 0.6^{\mathrm{a}} \\
7.4 \pm 0.1^{\mathrm{a}} \\
7.1 \pm 0.5^{\mathrm{a} * *}\end{array}$ & $\begin{array}{l}2.01 \\
1.78 \\
1.55 \\
\end{array}$ \\
\hline $\mathrm{EC}(\mathrm{ms} / \mathrm{cm})$ & $\begin{array}{c}\text { First year } \\
\text { Second year } \\
\text { Combined }\end{array}$ & $\begin{array}{l}0.09 \pm 0.05^{\mathrm{d}} \\
0.15 \pm 0.05^{\mathrm{d}} \\
0.12 \pm 0.04^{\mathrm{d}}\end{array}$ & $\begin{array}{c}0.63 \pm 0.04^{\mathrm{c}} \\
0.8 \pm 0.15^{\mathrm{c}} \\
0.71 \pm 0.14^{\mathrm{c}} \\
\end{array}$ & $\begin{array}{c}0.76 \pm 0.07^{\mathrm{c}} \\
1.37 \pm 0.07^{\mathrm{b}} \\
1.1 \pm 0.34^{\mathrm{b}}\end{array}$ & $\begin{array}{l}0.8 \pm 0.2^{\mathrm{bc}} \\
1.62 \pm 0.1^{\mathrm{a}} \\
1.2 \pm 0.5^{\mathrm{b}}\end{array}$ & $\begin{array}{c}0.97 \pm 0.16^{\mathrm{b}} \\
1.75 \pm 0.11^{\mathrm{a}} \\
1.36 \pm 0.4^{\mathrm{a}}\end{array}$ & $\begin{array}{c}1.34 \pm 0.2^{\mathrm{a} *} \\
1.85 \pm 0.1 \\
1.6 \pm 0.3^{\mathrm{a} *}\end{array}$ & $\begin{array}{l}0.58 \\
0.57 \\
0.35 \\
\end{array}$ \\
\hline $\mathrm{Ca}(\mathrm{c}$ & $\begin{array}{c}\text { First year } \\
\text { Second year } \\
\text { Combined }\end{array}$ & $\begin{array}{l}11.95 \pm 0.9^{\mathrm{c}} \\
11.14 \pm 0.3^{\mathrm{c}} \\
11.54 \pm 0.7^{\mathrm{c}}\end{array}$ & $\begin{array}{l}12.9 \pm 0.3^{\mathrm{bc}} \\
15.9 \pm 0.6^{\mathrm{b}} \\
14.4 \pm 1.7^{\mathrm{b}} \\
\end{array}$ & $\begin{array}{c}14.15 \pm 0.3^{\mathrm{ab}} \\
17.9 \pm 1.4^{\mathrm{ab}} \\
16.01 \pm 2.2^{\mathrm{ab}}\end{array}$ & $\begin{array}{c}15.12 \pm 1.8^{\mathrm{a}} \\
18.45 \pm 0.9^{\mathrm{a}} \\
16.8 \pm 2.2^{\mathrm{a}} \\
\end{array}$ & $\begin{array}{c}13.9 \pm 0.7^{\mathrm{ab}} \\
19.32 \pm 1.5^{\mathrm{a} *} \\
16.6 \pm 3.1^{\mathrm{a}} \\
\end{array}$ & $\begin{array}{c}14.9 \pm 1.0^{\mathrm{a} * *} \\
18.7 \pm 1.2^{\mathrm{a}} \\
16.8 \pm 2.2^{\mathrm{a} * *}\end{array}$ & $\begin{array}{c}3.17 \\
4.8 \\
4.4 \\
\end{array}$ \\
\hline $\mathrm{K}\left(\mathrm{cmol}^{+} / \mathrm{kg}\right)$ & $\begin{array}{c}\text { First year } \\
\text { Second year } \\
\text { Combined }\end{array}$ & $\begin{array}{c}0.6 \pm 0.05^{\mathrm{b}} \\
0.72 \pm 0.08^{\mathrm{d}} \\
0.64 \pm 0.1^{\mathrm{d}}\end{array}$ & $\begin{array}{c}1.75 \pm 0.7^{\mathrm{b}} \\
3.1 \pm 0.5^{\mathrm{c}} \\
2.4 \pm 0.9^{\mathrm{c}} \\
\end{array}$ & $\begin{array}{l}3.9 \pm 1.5^{\mathrm{a}} \\
5.3 \pm 0.6^{\mathrm{b}} \\
4.6 \pm 1.3^{\mathrm{b}}\end{array}$ & $\begin{array}{c}5.3 \pm 2.0^{\mathrm{a} * *} \\
7.4 \pm 1.0^{\mathrm{a}} \\
6.4 \pm 1.8^{\mathrm{a}}\end{array}$ & $\begin{array}{c}5.2 \pm 1.5^{\mathrm{a}} \\
7.8 \pm 1.0^{\mathrm{a}} \\
6.5 \pm 1.8^{\mathrm{a} *} \\
\end{array}$ & $\begin{array}{c}4.2 \pm 0.6^{\mathrm{a}} \\
8.3 \pm 1.1^{\mathrm{a} *} \\
6.2 \pm 2.3^{\mathrm{a}}\end{array}$ & $\begin{array}{c}4.63 \\
4.3 \\
3.8 \\
\end{array}$ \\
\hline $\left.\mathrm{nol}^{+} / \mathrm{kg}\right)$ & $\begin{array}{c}\text { First year } \\
\text { Second year } \\
\text { Combined }\end{array}$ & $\begin{array}{l}0.21 \pm 0.04^{\mathrm{c}} \\
0.24 \pm 0.05^{\mathrm{c}} \\
0.22 \pm 0.04^{\mathrm{b}}\end{array}$ & $\begin{array}{l}0.81 \pm 0.2^{\mathrm{b}} \\
0.6 \pm 0.08^{\mathrm{b}} \\
0.7 \pm 0.2^{\mathrm{a} *}\end{array}$ & $\begin{array}{c}0.5 \pm 0.09^{\mathrm{a}} \\
0.74 \pm 0.12^{\mathrm{ab}} \\
0.62 \pm 0.1^{\mathrm{a}}\end{array}$ & $\begin{array}{c}0.6 \pm 0.04^{\mathrm{ab}} \\
0.8 \pm 0.06^{\mathrm{ab}} \\
0.7 \pm 0.1^{\mathrm{a} *}\end{array}$ & $\begin{array}{c}0.45 \pm 0.1^{\mathrm{ac} *} \\
0.92 \pm 0.2^{\mathrm{a} *} \\
0.7 \pm 0.3^{\mathrm{a} *}\end{array}$ & $\begin{array}{c}0.43 \pm 0.03^{\mathrm{ac}} \\
0.83 \pm 0.2^{\mathrm{ab}} \\
0.6 \pm 0.2^{\mathrm{a}}\end{array}$ & $\begin{array}{c}0.6 \\
0.53 \\
0.39 \\
\end{array}$ \\
\hline $\mathrm{Mg}\left(\mathrm{cmol}^{+} / \mathrm{kg}\right)$ & $\begin{array}{c}\text { First year } \\
\text { Second year } \\
\text { Combined }\end{array}$ & $\begin{array}{c}3.99 \pm 0.9 \\
3.8 \pm 0.5^{b} \\
3.9 \pm 0.7\end{array}$ & $\begin{array}{c}5.4 \pm 1.5 \\
7.4 \pm 1.5^{\mathrm{a}} \\
6.4 \pm 1.8\end{array}$ & $\begin{array}{c}5.8 \pm 2.0 \\
8.7 \pm 0.6^{\mathrm{a}} \\
7.3 \pm 2.0\end{array}$ & $\begin{array}{c}5.0 \pm 2.8 \\
8.5 \pm 1.2^{\mathrm{a}} \\
6.8 \pm 2.7\end{array}$ & $\begin{array}{c}5.99 \pm 1.3 \\
7.9 \pm 2.2^{\mathrm{a} * *} \\
6.9 \pm 1.9\end{array}$ & $\begin{array}{c}4.8 \pm 1.1 \\
7.6 \pm 1.9^{\mathrm{a}} \\
6.2 \pm 2.0\end{array}$ & $\begin{array}{l}\text { NS } \\
4.75 \\
\text { NS }\end{array}$ \\
\hline $\mathrm{OC}(\%)$ & $\begin{array}{l}\text { First year } \\
\text { Second year } \\
\text { Combined }\end{array}$ & $\begin{array}{c}0.8 \pm 0.09^{\mathrm{d}} \\
0.77 \pm 0.1^{\mathrm{d}} \\
0.8 \pm 0.1^{\mathrm{d}}\end{array}$ & $\begin{array}{c}0.8 \pm 0.1^{\mathrm{d}} \\
1.4 \pm 0.15^{\mathrm{c}} \\
1.1 \pm 0.3^{\mathrm{c}}\end{array}$ & $\begin{array}{c}1.1 \pm 0.08^{\mathrm{c}} \\
1.9 \pm 0.07^{\mathrm{b}} \\
1.5 \pm 0.4^{\mathrm{b}}\end{array}$ & $\begin{array}{c}1.22 \pm 0.04^{\mathrm{c}} \\
2.2 \pm 0.12^{\mathrm{b}} \\
1.7 \pm 0.5^{\mathrm{b}}\end{array}$ & $\begin{array}{c}1.47 \pm 0.1^{\mathrm{b}} \\
2.8 \pm 0.2^{\mathrm{a} *} \\
2.1 \pm 0.7^{\mathrm{a}}\end{array}$ & $\begin{array}{c}1.71 \pm 0.1^{\mathrm{a} *} \\
2.6 \pm 0.1^{\mathrm{a}} \\
2.2 \pm 0.5^{\mathrm{a} *}\end{array}$ & $\begin{array}{l}0.42 \\
0.52 \\
0.63 \\
\end{array}$ \\
\hline Av. P (ppm) & $\begin{array}{c}\text { First year } \\
\text { Second year } \\
\text { Combined }\end{array}$ & $\begin{array}{c}13.6 \pm 1.9^{\mathrm{e}} \\
14.06 \pm 1.5^{\mathrm{c}} \\
13.8 \pm 1.5^{\mathrm{e}}\end{array}$ & $\begin{array}{c}24.2 \pm 1.5^{\mathrm{dc}} \\
58.1 \pm 2.5^{\mathrm{b}} \\
41.1 \pm 18.6^{\mathrm{d}}\end{array}$ & $\begin{array}{c}32.6 \pm 3.4^{\mathrm{c}} \\
59.8 \pm 2.9^{\mathrm{b}} \\
46.1 \pm 15.1^{\mathrm{cd}}\end{array}$ & $\begin{array}{c}38.8 \pm 9.4^{\mathrm{bc}} \\
62.7 \pm 5.0^{\mathrm{b}} \\
50.78 \pm 14.7^{\mathrm{c}}\end{array}$ & $\begin{array}{c}49.2 \pm 3.2^{\mathrm{b}} \\
79.7 \pm 2.8^{\mathrm{a}} \\
64.4 \pm 16.9^{\mathrm{b}}\end{array}$ & $\begin{array}{l}65.8 \pm 7.5^{\mathrm{a} *} \\
80.7 \pm 3.9^{\mathrm{a} *} \\
73.2 \pm 9.7^{\mathrm{a} *}\end{array}$ & $\begin{array}{l}24.9 \\
16.9 \\
13.6 \\
\end{array}$ \\
\hline $\operatorname{CEC~}\left(\mathrm{cmol}^{+} / \mathrm{kg}\right)$ & $\begin{array}{l}\text { First year } \\
\text { Second year } \\
\text { Combined }\end{array}$ & $\begin{array}{l}19.7 \pm 1.3^{b} \\
20.6 \pm 0.9^{d} \\
20.2 \pm 1.1^{c}\end{array}$ & $\begin{array}{l}21.1 \pm 0.9^{\mathrm{b}} \\
27.1 \pm 1.5^{\mathrm{c}} \\
24.1 \pm 3.4^{\mathrm{b}}\end{array}$ & $\begin{array}{l}24.9 \pm 2.1^{\mathrm{a}} \\
28.3 \pm 1.0^{\mathrm{b}} \\
26.6 \pm 2.4^{\mathrm{a}}\end{array}$ & $\begin{array}{l}24.8 \pm 1.4^{\mathrm{a}} \\
31.8 \pm 1.0^{\mathrm{a}} \\
28.4 \pm 3.9^{\mathrm{a}}\end{array}$ & $\begin{array}{c}25.6 \pm 0.9^{\mathrm{a} * *} \\
32.7 \pm 1.8^{\mathrm{a}} \\
29.2 \pm 4.0^{\mathrm{a} * *}\end{array}$ & $\begin{array}{c}24.6 \pm 2.5^{\mathrm{a}} \\
33.2 \pm 1.7^{\mathrm{a} * *} \\
28.9 \pm 5.0^{\mathrm{a}}\end{array}$ & $\begin{array}{l}5.9 \\
5.5 \\
4.8\end{array}$ \\
\hline
\end{tabular}

Within rows, means followed by the same letter are not significantly different $(p>0.05) ;{ }^{*}$ significant at $p \leq 0.01 ;{ }^{* *}$ significant at $p \leq 0.05$; NS, not significant; LSD, least significant difference; PB; parthenium biochar; SD, standard deviation.

TABLE 6: Multivariate analysis for the soil micronutrients contents related with the soil sample years and parthenium biochar dosages.

\begin{tabular}{|c|c|c|c|c|c|c|c|c|c|}
\hline \multirow{2}{*}{ Sources of variations } & \multirow{2}{*}{ DF } & \multicolumn{2}{|c|}{$\mathrm{Cu}(\mathrm{mg} / \mathrm{L})$} & \multicolumn{2}{|c|}{$\mathrm{Zn}(\mathrm{mg} / \mathrm{L})$} & \multicolumn{2}{|c|}{$\mathrm{Mn}(\mathrm{mg} / \mathrm{L})$} & \multicolumn{2}{|c|}{$\mathrm{Fe}(\mathrm{mg} / \mathrm{L})$} \\
\hline & & MS & $\mathrm{P}$ & MS & $\mathrm{P}$ & MS & $\mathrm{P}$ & MS & $\mathrm{P}$ \\
\hline $\begin{array}{l}\text { SSY } \\
\end{array}$ & 1 & 0.94 & $p<0.05$ & 0.02 & $p>0.05$ & 5.4 & $p \leq 0.001$ & 14.7 & $p<0.05$ \\
\hline PBD & 5 & 1.67 & $p \leq 0.001$ & 0.69 & $p \leq 0.001$ & 3.5 & $p \leq 0.001$ & 45.6 & $p \leq 0.001$ \\
\hline $\mathrm{SSY} * \mathrm{PBD}$ & 5 & 0.19 & $p>0.05$ & 0.007 & $p>0.05$ & 0.33 & $p<0.05$ & 0.75 & $p>0.05$ \\
\hline Error & 24 & 0.12 & - & 0.018 & - & 0.1 & - & 2.9 & - \\
\hline Mean & 0.42 & - & 0.74 & - & 2.4 & - & 8.1 & - & - \\
\hline$R$-square $\left(R^{2}\right)$ & 0.78 & - & 0.89 & - & 0.90 & - & 0.77 & - & - \\
\hline
\end{tabular}

SSY, soil sample years; PBD, parthenium biochar dosages; MS, mean square; P, probability level; DF, degree of freedom.

micronutrients with increasing parthenium biochar dosages from $12.5 \mathrm{t} /$ ha to $23 \mathrm{t} /$ ha (Figure 1). However, the highest average values of $\mathrm{Fe}(6.0 \pm 0.6), \mathrm{Cu}(0.96 \pm 0.5), \mathrm{Mn}$ $(3.2 \pm 0.3)$, and $\mathrm{Zn}(1.3 \pm 0.2)$ registered in soils amended with no biochar (Figure 1).

3.3. Variation of the Soil Chemical Properties with Integrated Treatments. The amendment potential of different dosages of biochar on the chemical properties of acidic soils in the two years' time was evaluated using multivariate analysis. PCA axis 1 accounted for $74.34 \%$ variance and total eigenvalue equivalence to 10.407 and positively correlated with the soil EC (0.294), CEC (0.291), and available phosphorus (0.289) and negatively loading with the micronutrients including $\mathrm{Mn}(-0.27)$ and $\mathrm{Zn}(-0.25)$ (Table 7; Figure 2). The PCA biplot revealed the soil sampling years (first and second) and biochar dosages. Thus soil sample year 


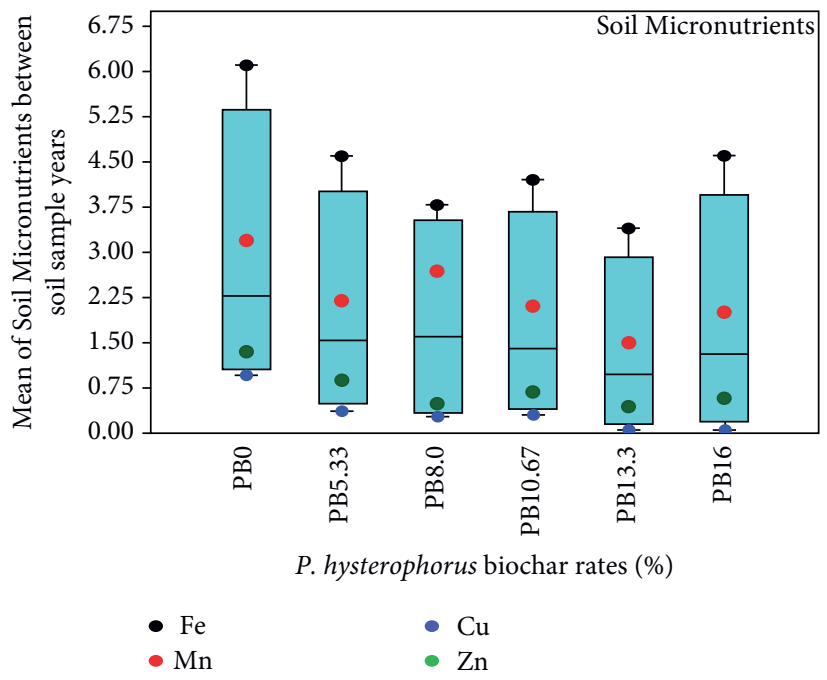

FIgURE 1: The effect of $P$. hysterophorus biochar on soil micronutrients of acidic soil between two soil sampling years.

TABLE 7: Eigenvector coefficients for the soil chemical properties contained in the first sixth principal components (PC1-6) derived from the principal component analysis of the indicated parameters in six treatments over two years.

\begin{tabular}{|c|c|c|c|c|c|c|}
\hline Soil parameters & PC1 & PC2 & PC3 & PC4 & PC5 & PC6 \\
\hline $\mathrm{pH}\left(\mathrm{H}_{2} \mathrm{O}\right)$ & 0.285 & 0.0163 & -0.251 & 0.215 & 0.018 & 0.313 \\
\hline $\mathrm{pH}(\mathrm{KCl})$ & 0.282 & -0.0028 & -0.252 & 0.238 & 0.039 & 0.329 \\
\hline $\mathrm{EC}$ & 0.294 & 0.012 & 0.217 & 0.013 & 0.024 & 0.108 \\
\hline Av. P & 0.289 & -0.0176 & 0.222 & 0.131 & -0.027 & -0.114 \\
\hline OC & 0.282 & 0.159 & 0.332 & 0.062 & -0.135 & 0.050 \\
\hline CEC & 0.291 & 0.252 & 0.109 & 0.081 & 0.043 & -0.126 \\
\hline $\mathrm{Ca}^{++}$ & 0.283 & 0.328 & 0.037 & 0.046 & 0.126 & -0.0014 \\
\hline $\mathrm{K}^{+}$ & 0.272 & -0.005 & 0.428 & 0.039 & 0.054 & 0.154 \\
\hline $\mathrm{Na}^{+}$ & 0.224 & 0.109 & 0.0024 & -0.880 & 0.266 & -0.005 \\
\hline $\mathrm{Mg}^{++}$ & 0.224 & 0.449 & -0.631 & -0.0271 & -0.090 & -0.133 \\
\hline $\mathrm{Cu}$ & -00.236 & 0.443 & 0.171 & -0.172 & -0.545 & 0.512 \\
\hline $\mathrm{Zn}$ & -00.251 & 0.463 & 0.133 & 0.101 & -0.165 & -0.398 \\
\hline $\mathrm{Mn}$ & -0.271 & 0.059 & -0.0471 & -0.022 & 0.331 & 0.537 \\
\hline $\mathrm{Fe}$ & -0.239 & 0.418 & 0.151 & 0.223 & 0.667 & 0.007 \\
\hline Eigen value & 10.407 & 0.811 & 0.677 & 0.537 & 0.417 & 0.314 \\
\hline$\%$ variance & 0.743 & 0.058 & 0.048 & 0.038 & 0.03 & 0.022 \\
\hline
\end{tabular}

Bold values indicate the most and the least explained variables.

two has a high degree of association with the biochar dosages, apart from the control treatments that had no significant changes across soil sampling years owning to no parthenium biochar (Figure 2). In contrast, the soil sample year one and biochar dosages did not show a higher association. However, the availability of those parthenium biochar-induced nutrients enhanced along with years within decreasing trends of the micronutrients.

3.4. Effect of Biochar on the Agronomic Performance of Wheat Varieties. The multivariate analysis revealed that plant height $(\mathrm{PH})$ and head length (HL) of wheat varieties were significantly $(p \leq 0.01 ; p \leq 0.05)$ affected by the application of different biochar during the first and second years growth period (Table 8). There were significant differences $(p \leq 0.001)$ in seed number per plant (SNPP) and spikelet numbers (SN) among the wheat varieties between biochar amendments across both years (Table 8). Despite the influences in the biochar rates, there was no interaction effect among the treatment factors. The analysis further indicated that Ogolcha (AGO) wheat variety showed the highest performance in $\mathrm{PH}(62.67 \pm 4.6 \mathrm{~cm})$, HL $(6.5 \pm 0.4 \mathrm{~cm})$, SNPP $(28.33 \pm 1.3)$, and SN $(29.0 \pm 2.9)$ in between 12.5 and $19.5 \mathrm{t} /$ ha biochar dosages followed by the Kekeba (KEK) wheat variety (Figure 3). However, the Kingbird (KIN) variety had comparatively lower growth performance due to parthenium biochar additions in two growing years (Figure 3).

Root length, root dry biomass, and shoot dry biomass of wheat varieties also showed significant changes $(p \leq 0.001)$ between biochar dosages and wheat growing periods. Correspondingly, there was a considerable interaction effect between the concentration of biochar and wheat varieties in root length 

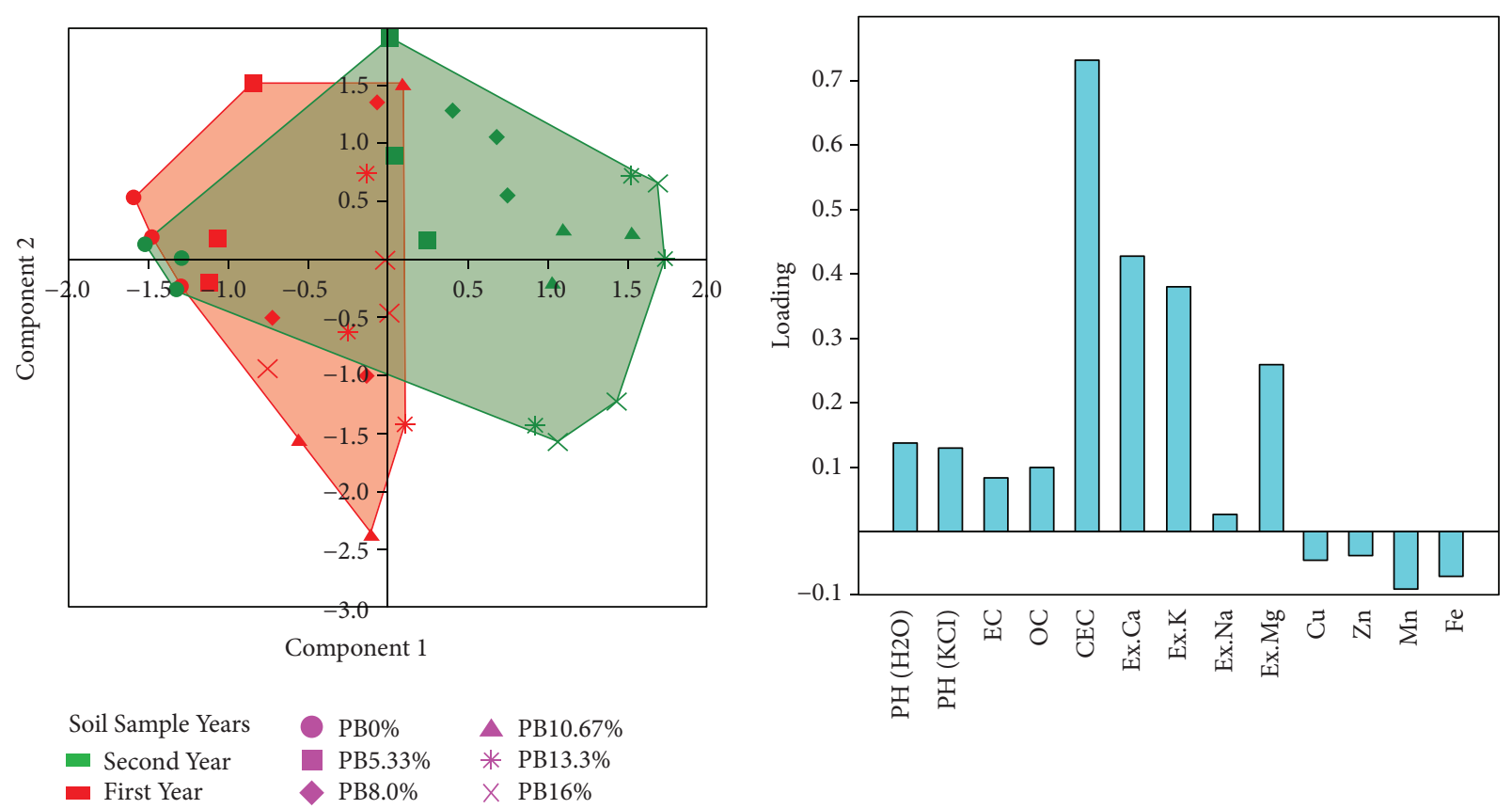

Figure 2: Biplot PCA chart indicating soil chemical properties of acidic soil as influenced by the parthenium biochar across years. The soil characteristics used were pH, EC, \% OC, CEC, Ex.Ca, Ex.K, Ex.Mg, Ex.Mg, Cu, Zn, Mn, and Fe.

and root dry biomass) and shoot dry biomass (Table 8). However, the combined factors (biochar rates, wheat varieties, and cropping years) have no significant interaction effect on the agronomic parameters (Table 8). Wheat varieties KEK and AGO were comparatively higher overall average values in terms of SDB (12.0; 11.47), RDB (5.03; 5.0), and RL (35.5; 43.75), respectively, compared to the KIN wheat variety across the two cropping years (Figure 4).

\section{Discussion}

4.1. Effect of Biochar Addition on the Soil Physical Properties. The results of the study showed that an increase in the applied biochar dosage increased the clay content and decreased the sand fraction. Van-Zwieten et al. [22] demonstrated that biochar applied in the soil mixture could significantly influence soil texture and increase the clay fractions. However, there was no considerable difference across years and with the combined effect of the variables, which could be associated with the nature of parent material that cannot modify in two years. The notable difference in SMC of acidic soils was the result of biochar application that enhances the formation of large surface area and increases soil porosity, which increases the water holding capacity. This finding agreed with the work of Uzoma et al. [34], who stated that the amendment of soil by cow manure biochar lifts up SMC by $15 \%$. Similarly, Asai et al. [35] and Jones et al. [36] reported the significant effect of biochar to increase the water holding capacity of degraded soils.

4.2. Effect of P. hysterophorus Biochar on the Chemical Characteristics of Acidic Soils. Biochar is known to decrease soil acidity and, in turn, enhance plant growth by increasing soil fertility [37]. Thus, in all treatments, application of $P$. hysterophorus biochar increased the soil $\mathrm{pH}$ by shifting from acidic (5.4) to neutral (6.7) and slightly alkaline (7.8) conditions (Table 5), which is an ideal soil $\mathrm{pH}$ environment for crop growth. The effect was due to the biochar having higher $\mathrm{pH}$ content (Table 1) and, in turn, a liming function capable of neutralizing acid-forming cations in the soil exchange sites [38] and the dissolution of alkaline carbonates and hydroxides minerals present mainly in the ash fraction of the biochar [12]. Previous studies on the rice husk biochar increased the acidic tea garden from soil $\mathrm{pH} 3.33$ to 3.63 [39]; the chicken manure biochar indicated a considerable change in the soil pH from 3.9 to 5.1 [40], and when $39 \mathrm{t}^{\mathrm{h}} \mathrm{ha}^{-1}$, herbaceous feedstock applied in the soil alter the $\mathrm{pH}$ from 7.1 to 8.1 [17]. Due to the chemical composition of the char (Table 1), the soil EC has augmented within the biochar rates. However, irrespective of the EC values, the effect of salinity was found negligible in all soil types (Table 5, [41]). Mensah and Frimpong [42] indicated that the soil EC was increased by $10.8 \%$ in the $2.5 \mathrm{t} / \mathrm{ha}$ of tannery wastes biochar treatment. Despite these enhancing results, there is a study that reports the opposite for long-term field trials. Jones et al. [36] showed that three years of biochar addition to the soil system significantly $(p<0.05)$ reduced the soil EC from 46 to $43 \mu \mathrm{s} \cdot \mathrm{cm}^{-1}$ in a UK field trial. $P$. hysterophorus biochartreated soils found in the ranges of between medium to a very high content of the exchangeable base and CEC [41]. These higher contents are ascribed to the potential effect of the pyrolysis carbon to release base-forming cations, which offset the incidence of soil acidity. Similar findings were reported by Agegnehu et al. [17], Agegnehu et al. [27], and Mensah and Frimpong [42] who found a highly variable charge in organic material that increases the exchangeable 


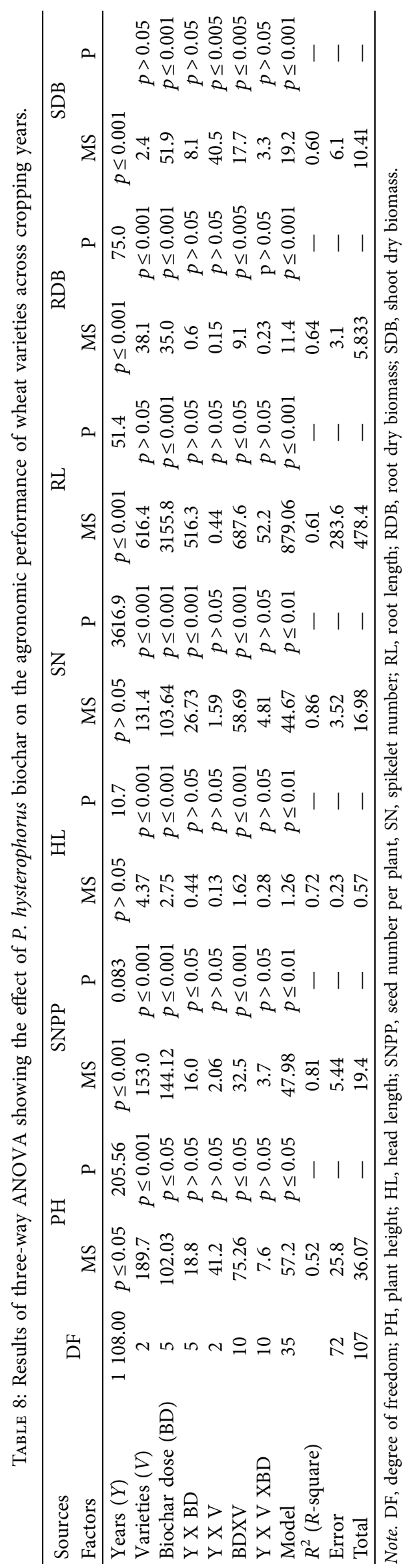




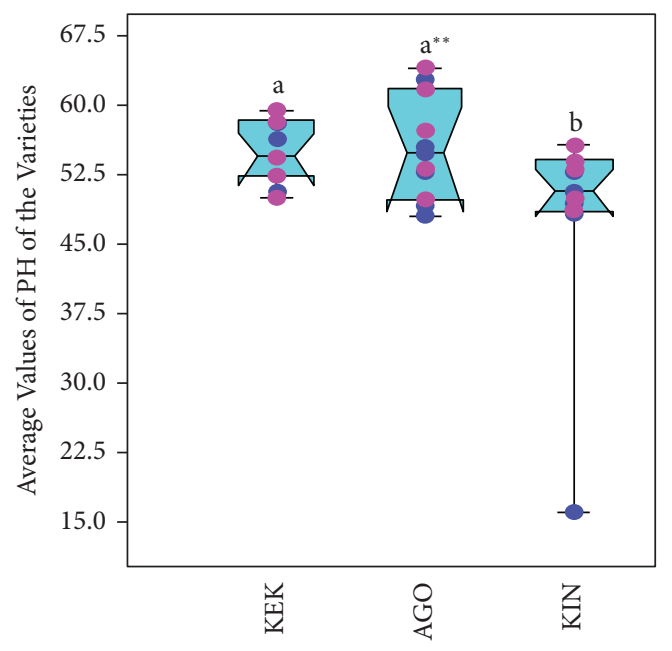

- First Year

- Second Year

(a)

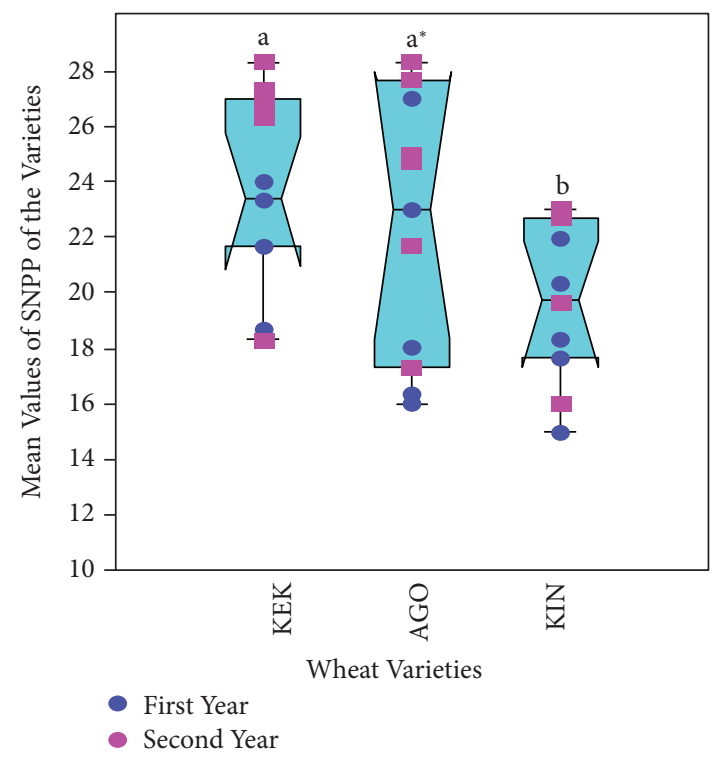

(c)

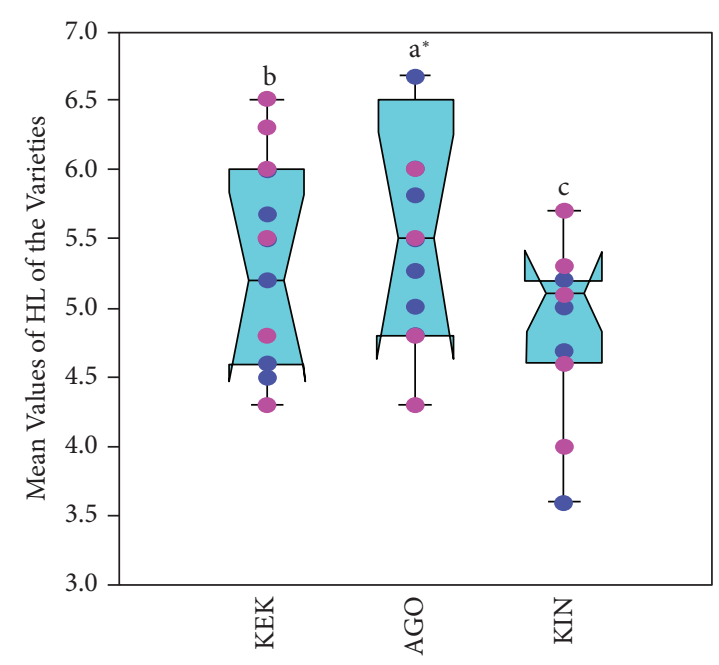

- First Year

- Second Year

(b)

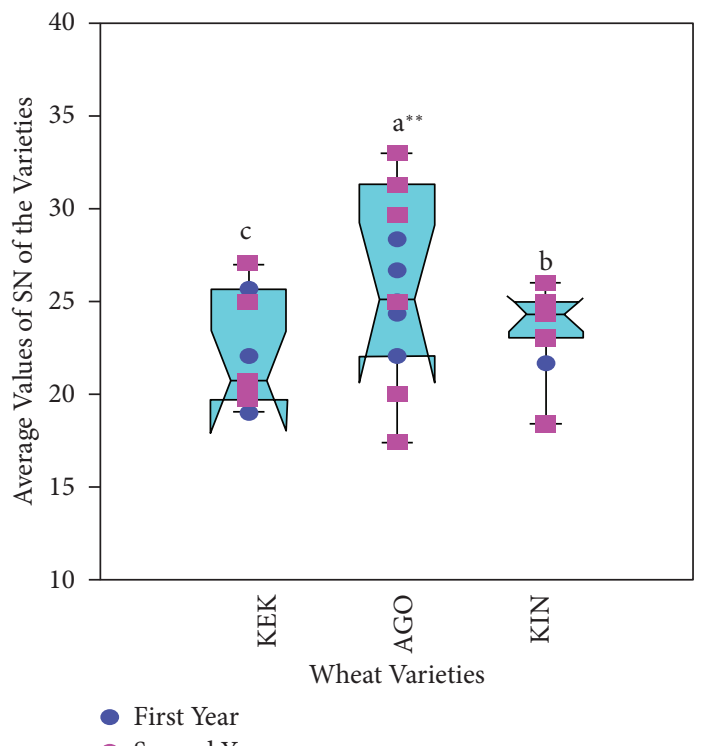

(d)

FIGURe 3: The agronomic performance of wheat varieties (KEK, AGO, and KIN) as influenced by different parthenium biochar and wheat growing years.

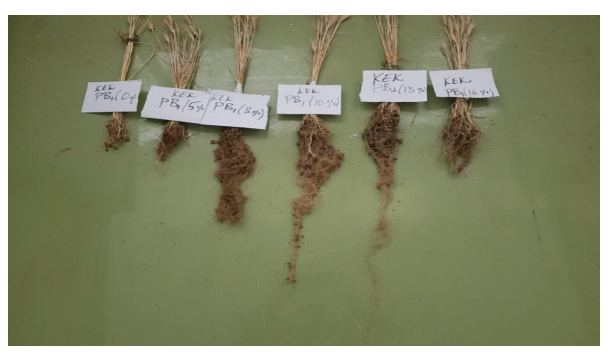

FIGURE 4: The root length of Kekeba wheat variety with increasing the biochar.

bases after the addition of biochar. Similar findings of an increase in soil CEC (from 7.41 to $10.8 \mathrm{cmol}^{+} / \mathrm{kg}$ ) and PBS (6.4 to $26 \%$ ) in the biochar-treated soils were also reported by Bhattarai et al. [43]. Also, an increasing trend in $\mathrm{Mg}$ content compared to the control treatment, but with no considerable difference was observed in this study. And 
this might be related to the lower production temperature and the feedstock nature. In the same ways, no significant difference in the concentration of $\mathrm{Mg}$ content was due to the application of red oak biochar $\left(500^{\circ} \mathrm{C} ; 2 \%\right)$ reported by Mensah and Frimpong [42]. On the other hand, the addition of $P$. hysterophorus biochar (12.5-26.5 $\mathrm{t} / \mathrm{ha})$ to acidic soil improved Av. P and SOC contents by 5.5 and $2.9 \%$, respectively, compared to the control (Table 5 ). This trend of an increase in Av. P and SOC contents are associated with the effects of biochar application. The result agreed with the findings of Jones et al. [36], who reported the effect of biochar in the improvement of total organic carbon from 2.27 to $2.78 \%$ and Av. P from 15.7 to $15.8 \mathrm{mg} \cdot \mathrm{kg}^{-1}$. This was due to the biochar effect that increased the soil $\mathrm{pH}$ (Table 1) and surface area of soil colloids, which in turn increased the nutrient holding capacity of the soil. In similar studies, Agegnehu et al. [17] and Mensah and Frimpong [42] also found higher soil Av. P in biochar-amended soil due to an increase in soil $\mathrm{pH}$.

4.3. Potential of Parthenium Biochar to Immobilize Soil Micronutrients. The availability of $\mathrm{Fe}, \mathrm{Mn}, \mathrm{Zn}, \mathrm{Cu}$, and $\mathrm{Zn}$ is generally higher at lower $\mathrm{pH}$ or acidic soil [44]. However, in this study, the addition of parthenium biochar in acidic soils reduced the bioavailability of micronutrients ( $\mathrm{Fe}, \mathrm{Mn}, \mathrm{Cu}$, and $\mathrm{Zn}$ ) because the application of biochar in acidic soil can adsorb and immobilize heavy metals $[11,15]$. Also, as $P$. hysterophorus biochar dosages increase, rates of micronutrients significantly decrease in the soil system. As a result, the proportion of heavy metals in biochar-treated soils was found in the range of low to medium compared with control as per the ratings of FAO [41]. In a pot trial study, the application of hardwoodderived biochar on contaminated soils also reduced the availability of zinc ( $\mathrm{Zn})$ and cadmium $(\mathrm{Cd})$ metal types [13]. Similarly, Qiao et al. [16] described that the application of biochar in acidic soils significantly decreased acid-soluble heavy metal concentrations ( $\mathrm{Fe}, \mathrm{Mn}, \mathrm{Pb}$, and $\mathrm{Cd}$ ) and their bioavailability for plants. Moreover, eigenvector coefficients and the PCA biplot (Table 7; Figure 2) showed a strong and positive association between the biochar dosages and years in improving essential soil nutrients ( $\mathrm{pH}$, basic cations, Av. P, and SOC), but micronutrients were negative loading with increasing biochar dosages and soil sampling years. Previous studies by Agegnehu et al. [27] indicated that most of the parameters (K, SOC, and CEC) equally contributed to the total variation and thus positive loading.

4.4. Performance of Wheat Varieties Influenced by P. hysterophorus Biochar. The application of biochar in the acidic soil exhibited a greater effect on plant height $(\mathrm{PH})$ and head length (HL), especially in AGO and KEK wheat varieties as compared to the KIN variety (Figure 3 ). In contrast, the control treatment had to wane the agronomic performance of the studied wheat varieties, which may be due to the soil minerals are being chemically less available to the plants in acidic soil. But the higher growth performance in biochar-amended soils is the capacity of the char to lower soil acidity and improves the potential availability of essential nutrients for wheat growth. And this was in line with the findings of Mensah and Frimpong [42] who reported the increments of plant height, the number of leaves, and stems girth of two maize varieties after the application of corncob biochar alone or in combined with compost in the acidic rainforest and coastal savannah soils. However, the performances of the varieties decreased as the parthenium biochar dosages increased above $23 \mathrm{t} / \mathrm{ha}$, which could be due to an increase in soil $\mathrm{pH}(>7.0)$ above the optimum level where wheat growth requires a $\mathrm{pH}$ of 6 to 7 . A previous study by Asai et al. [35] revealed that the addition of higher than $16 \mathrm{t} / \mathrm{ha}$ of teak and rosewood biochar in the field condition of acidic soil reduces rice growth. The $P$. hysterophorus biochar significantly increases the radicle length, root dry biomass, shoot dry biomass, seed number per plant (SNPP), and spikelet number (SN) of the wheat varieties, which might be due to liming effect of basic cations available in the char (Table 1). An increasing trend in biomass agrees with the findings of Agegnehu et al. [17] and Bhattarai et al. [42]. However, Van-Zwieten et al. [22] observed that the wheat and soybean biomass production was not affected solely by the slow pyrolysis of paper mill biochar addition. Moreover, in this study, the AGO wheat variety observed the higher entire biomass in acidic soil amended by parthenium biochar, followed by the KEK variety, whereas the lower entire growth performance was recorded in KIN wheat variety with a similar amendment in acidic soils.

\section{Conclusions and Future Perspectives}

Soil acidity and invasive weeds significantly reduced crop productivity and sustainability. Thus, ameliorating soil acidity through averting noxious weed into a usable form of biochar is indispensable to boost fertility and crop productivity. The present study revealed that P. hysterophorus biochar considerably increased the SMC, $\mathrm{pH}, \mathrm{Av}$. P, exchangeable bases, and SOC compared to the control treatments (with no biochar). However, soil particle size fractions did not show significant variation across the years. P. hysterophorus biochar significantly decreased the bioavailability of metal contaminants that attributes to the occurrence of soil acidity. Furthermore, the higher agronomic performance of the wheat varieties found in the biochar-amended acidic soils. Also, the higher crop assay parameters were recorded in Ogolcha (AGO) and Kekeba (KEK) wheat varieties compared to the lower performance of the Kingbird (KIN) variety. However, the performances of the entire wheat varieties gradually declined as the concentration of chars exceeded over $23 \mathrm{t} / \mathrm{ha}$. To this end, integrated agricultural inputs with the $P$. hysterophorus biochar are very crucial to amend soil acidity. More comprehensive studies will be needed to evaluate the effectiveness of $P$. hysterophorus biochar in the amelioration of acidic soil, soil 
microorganisms, and yields on field conditions of different soil types.

\section{Data Availability}

The raw data sets used and/analyzed during the current study are available from the corresponding author on reasonable request.

\section{Conflicts of Interest}

The authors declare that there are no conflicts of interest.

\section{Authors' Contributions}

MM has designed the project and contributed to data collection, laboratory analysis, interpretation, and manuscript write-up. EM was involved in data analysis, manuscript organization, and edition. SM and ES guided, edited the study, and critically reviewed the ideas. AH was involved in data collection and laboratory analysis. All authors read and approved the final manuscript.

\section{Acknowledgments}

The authors are thankful to the farmers, Holeta Agricultural Institute Soil Laboratory Department and Gonder Soil Testing technicians, and the anonymous reviewers of the Amhara Regional Agricultural Research Institute soil department for their valuable comments. The collection of the data and laboratory expenses herein were supported by Woldia University Research and Community Service office annual grant fund.

\section{Supplementary Materials}

Supplementary 1: framework of the P. hysterophorus biochar preparation. Supplementary 2: conceptual framework of the soil sample preparation and layout of the research design for cropping. Supplementary Materials

\section{References}

[1] IFPRI, Constraints and Opportunities for Enhancing the System, Fertilizer and Soil Fertility Potential in Ethiopia, Washington, WA, USA, 2010.

[2] EATA, Status of Soil Resources in Ethiopia and Priorities for Sustainable Management, GSP for Eastern and Southern Africa, Nairobi, Kenya, 2013.

[3] T. Seta, A. Assefa, F. Mesfin, and A. Balcha, "Distribution status and the impact of parthenium weed (P. hysterophorus L.) at Gedeo zone (Southern Ethiopia)," African Journal of Agricultural Research, vol. 8, no. 4, pp. 386-397, 2013.

[4] A. Javaid, S. Shafique, R. Bajwa, and S. Shafique, "Effect of aqueous extracts of allelopathic crops on germination and growth of Parthenium hysterophorus L," South African Journal of Botany, vol. 72, no. 4, pp. 609-612, 2006.

[5] T. Tamado and P. Milberg, "Control of parthenium (Parthenium hysterophorus) in grain Sorghum (Sorghum bicolor) in the smallholder farming system in eastern Ethiopial," Weed Technology, vol. 18, no. 1, pp. 100-105, 2004.
[6] W. Mersie and M. Singh, "Allelopathic effect of parthenium (Parthenium hysterophorus L.) extract and residue on some agronomic crops and weeds," Journal of Chemical Ecology, vol. 13, no. 7, pp. 1739-1747, 1987.

[7] A. Khalq, F. Aslam, F. Matloob et al., "Phytotoxic activity of parthenium against wheat and canola differ with plant parts and bioassays techniques," Planta Daninha, vol. 34, no. 1, pp. 11-24, 2016.

[8] T. Tefera, "Allelopathic effects of Parthenium hysterophorus extracts on seed germination and seedling growth of Eragrostis tef," Journal of Agronomy and Crop Science, vol. 188, no. 5, pp. 306-310, 2002.

[9] W. Ahmad, S. Hussain, W. Malik et al., "Parthenium weeds compost: an environment friendly way of its control and to harness its allelopathic effect," Pakistan Journal of Weed Science Research, vol. 21, no. 4, pp. 505-516, 2015.

[10] S. A. Kumar, P. Tak, R. Ameta, and S. C. Ameta, "Effect of compost of Parthenium hysterophorus on growth and yield of wheat (Triticum aestivum): a comparative study," American Journal of Science, vol. 12, no. 10, pp. 34-37, 2016.

[11] X. Cao and W. Harris, "Properties of dairy-manure-derived biochar pertinent to its potential use in remediation," Bioresource Technology, vol. 101, no. 14, pp. 5222-5228, 2010.

[12] J. Lehmann, M. C. Rillig, J. Thies, C. A. Masiello, W. C. Hockaday, and D. Crowley, "Biochar effects on soil biota-a review," Soil Biology and Biochemistry, vol. 43, no. 9, pp. 1812-1836, 2011.

[13] L. Beesley, E. Moreno-Jiménez, J. L. Gomez-Eyles, E. Harris, B. Robinson, and T. Sizmur, "A review of biochars' potential role in the remediation, revegetation and restoration of contaminated soils," Environmental Pollution, vol. 159, no. 12, pp. 3269-3282, 2011.

[14] J. Lehmann, C. Czimczik, C. Laird, and S. Sohi, "Stability of biochar in soil," in Biochar for Environmental Management. Science and Technology, J. Lehmann and S. Josep, Eds., Earthscan, London, UK, 2009.

[15] M. Ahmad, A. U. Rajapaksha, J. E. Lim et al., "Biochar as a sorbent for contaminant management in soil and water: a review," Chemosphere, vol. 99, pp. 19-33, 2014.

[16] Y. Qiao, D. Crowley, K. Wang, H. Zhang, and H. Li, "Effects of biochar and Arbuscular mycorrhizae on bioavailability of potentially toxic elements in an aged contaminated soil," Environmental Pollution, vol. 206, no. 1, pp. 636-643, 2015.

[17] G. Agegnehu, A. M. Bass, P. N. Nelson, and M. I. Bird, "Benefits of biochar, compost and biochar-compost for soil quality, maize yield and greenhouse gas emissions in a tropical agricultural soil," The Science of the Total Environment, vol. 543, no. 1, pp. 295-306, 2016.

[18] T. Lang, A. D. Jensen, and P. A. Jensen, "Retention of organic elements during solid fuel pyrolysis with emphasis on the peculiar behavior of nitrogen," Energy and Fuels, vol. 19, no. 4, pp. 1631-1643, 2005.

[19] B. Liang, J. Lehmann, D. Solomon et al., "Black carbon increases cation exchange capacity in soils," Soil Science Society of America Journal, vol. 70, no. 5, pp. 1719-1730, 2006.

[20] P. G. Oguntunde, M. Fosu, A. E. Ajayi, and N. van de Giesen, "Effects of charcoal production on maize yield, chemical properties and texture of soil," Biology and Fertility of Soils, vol. 39, no. 4, pp. 295-299, 2004.

[21] F. G. A. Verheijen, S. Jefferey, A. C. Bastos, M. Vander-Velde, and I. Diafas, "Biochar application to soils-a critical scientific review of effects on soil properties, processes and functions," JRC Scientific and Technical Report EUR 24099 EN, Office for 
the Official Publications of the European Communities, Luxembourg, Europe, 2009.

[22] L. Van-Zwieten, S. Kimber, S. Morris et al., "Effects of biochar from slow pyrolysis of peppermill waste on agronomic performance and soil fertility," Plant and Soil, vol. 327, no. 1-2, pp. 235-246, 2010.

[23] Amhara regional state kombolcha district meteorology station (ARSKMS), database for metrological data source (2009-2019) of Gubalafto district, North Wollo, Ethiopia, 2020.

[24] D. Roopa, "Preparation of activated carbon from bitter orange peel (Citrus aurantium) and preliminary studies on its characteristics," International Journal of Engineering Research, vol. 7, no. 4, pp. 40-44, 2016.

[25] R. E. Masto, M. A. Ansari, J. George, V. A. Selvi, and L. C. Ram, "Co-application of biochar and lignite fly ash on soil nutrients and biological parameters at different crop growth stages of Zea mays," Ecological Engineering, vol. 58, no. 1, pp. 314-322, 2013.

[26] G. Han, J. Lan, Q. Chen, C. Yu, and S. Bie, "Response of soil microbial community to application of biochar in cotton soils with different continuous cropping years," Scientific Reports, vol. 7, no. 1, pp. 10184-10211, 2017.

[27] G. Agegnehu, M. I. Bird, P. N. Nelson, and A. M. Bass, "The ameliorating effects of biochar and compost on soil quality and plant growth on a ferralsol," Soil Research, vol. 53, no. 1, pp. 1-12, 2015.

[28] L. P. Van Reeuwijk, Procedures for Soil Analysis, International Soil Reference and Information Center (ISRIC), Wageningen, Netherlands, 3rd edition, 1992.

[29] ICARDA, Methods of Soil, Plants, and Water Analysis: A Manual for West Asia and Nort Africa Regions, International Center for Agricultural Research in the Dry Areas (ICARDA), Beirut, Lebanon, 2013.

[30] A. Walkeley and I. Black, An Examination of Degtjareff Methods for Determining Soil Organic Matter and a Proposed Modification of the Chromic Acid Titration Method, Wiley-Inter Science Publication, New York, NY, USA, 1934.

[31] W. L. Lindsay and W. A. Norvell, "Development of a DTPA soil test for zinc, iron, manganese, and copper," Soil Science Society of America Journal, vol. 42, no. 3, pp. 421-428, 1978.

[32] H. D. Chapman, "Cation exchange capacity," in Methods of Soil Analysis, C. A. Black, Ed., American Society of Agronomy, Madison, 1965.

[33] A. W. Anderson and J. R. Garlinge, The Wheat Book: Principle and Practice, Agriculture and Food, Australia, 2nd edition, 2000.

[34] K. C. Uzoma, M. Inoue, H. Andry, H. Fujimaki, A. Zahoor, and E. Nishihara, "Effect of cow manure biochar on maize productivity under sandy soil condition," Soil Use and Management, vol. 27, no. 2, pp. 205-212, 2011.

[35] H. Asai, B. K. Samson, H. M. Stephan et al., "Biochar amendment techniques for upland rice production in Northern Laos: soil physical properties, leaf SPAD and grain yield," Field Crops Research, vol. 111, no. 1-2, pp. 81-84, 2009.

[36] B. E. H. Jones, R. J. Haynes, and I. R. Phillips, "Effect of amendment of bauxite processing sand with organic materials on its chemical, physical and microbial properties," Journal of Environmental Management, vol. 91, no. 11, pp. 2281-2288, 2010.

[37] R. Shetty and N. B. Prakash, "Effect of different biochars on acid soil and growth parameters of rice plants under aluminium toxicity," Scientific Reports, vol. 10, pp. 12249-12310, 2020.

[38] J. L. Deenik, A. Diarra, G. Uehara, S. Campbell, Y. Sumiyoshi, and M. J. Antal, "Charcoal ash and volatile matter effects on soil properties and plant growth in an acid ultisol," Soil Science, vol. 176, no. 7, pp. 336-345, 2011.

[39] Y. Wang, R. Yin, and R. Liu, "Characterization of biochar from fast pyrolysis and its effect on chemical properties of the tea garden soil," Journal of Analytical and Applied Pyrolysis, vol. 110, no. 1, pp. 375-381, 2014.

[40] S. D. Joseph, M. Camps-Arbestain, Y. Lin et al., "An investigation into the reactions of biochar in soil," Australian Journal of Soil Research, vol. 48, no. 6-7, pp. 501-515, 2010.

[41] FAO, "Guide to laboratory establishment for plant nutrient analysis," in Fertilizer and Plant Nutrition BulletinFood and Agriculture Organization, Roma, Italy, 2008.

[42] A. K. Mensah and K. A. Frimpong, "Biochar and/or compost applications improve soil properties, growth, and yield of maize grown in acidic rainforest and coastal savannah soils in Ghana," International Journal of Agronomy, vol. 2018, Article ID 6837404, 8 pages, 2018.

[43] B. Bhattarai, J. Neupane, S. P. Dhakal et al., "Effect of biochar from different origin on physio-chemical properties of soil and yield of garden pea (Pisum sativum L.) at Paklihawa, Rupandehi, Nepal," World Journal of Agricultural Research, vol. 3, no. 4, pp. 129-138, 2015.

[44] A. Mccauley, C. Jones, and J. Jacobsen, "Soil pH and organic matter," Nutrient Management Module, vol. 8, 2009. 